\title{
Comparative efficacy of two fipronil spot-on formulations against experimental tick infestations (Ixodes ricinus) in dogs
}

\author{
Stéphane Bonneau • Sandeep Gupta • \\ Marie-Christine Cadiergues
}

Received: 17 March 2010 /Accepted: 21 May 2010 /Published online: 17 June 2010

(C) The Author(s) 2010. This article is published with open access at Springerlink.com

\begin{abstract}
A parallel-group-design, randomized, unicentre and blinded controlled study was undertaken to assess the efficacy of a new fipronil-based spot-on formulation applied once to dogs against experimental Ixodes ricinus infestations. Six dogs served as negative controls (group 1), six dogs served as positive controls (group 2) receiving the original fipronil spot-on (Frontline ${ }^{\circledR}$ spot-on Dog, Merial) at a dosage of $0.67 \mathrm{~mL}$ for a dog weighing from 2 to $10 \mathrm{~kg}$ and $1.34 \mathrm{~mL}$ for a dog weighing from 10.1 to $20 \mathrm{~kg}$ and six dogs were treated with a $10 \% \mathrm{w} / v$ fipronil-based spot-on solution (Effipro ${ }^{\circledR}$ Spot-on, Virbac SA) at an identical dosage (group 3, $0.67 \mathrm{~mL}$ for a dog weighing from 2 to 10 $\mathrm{kg}$ and $1.34 \mathrm{~mL}$ for a dog weighing from 10.1 to $20 \mathrm{~kg}$ ). Each dog was sedated and subsequently infested with 50 unfed adult $I$. ricinus on days $-7,-2,7,14,21,28$ and 35. Forty-eight hours after the treatment and $48 \mathrm{~h}$ after each challenge (days $-5,2,9,16,23,30$ and 37 ), the population of the remaining ticks was assessed for each animal. Geometric mean tick counts obtained were reduced by $99 \%$ and $94 \%$ on day 2 in groups 2 and 3, respectively,
\end{abstract}

\section{S. Bonneau}

Virbac S.A. Preclinical and Clinical Unit,

5ème avenue, BP 27, 06511 Carros, France

\section{S. Gupta}

Charles River Laboratories Preclinical Services Ireland Ltd,

Glenamoy Co.

Mayo, Ireland

\section{M.-C. Cadiergues $(\bowtie)$}

Dermatology, Groupe de Recherche des Animaux de Compagnie,

National Veterinary School,

BP 87614 23, chemin des Capelles,

31076 Toulouse, France

e-mail: mc.cadiergues@envt.fr compared to the negative control group. Dogs were protected from re-infestations with an efficacy of $>90 \%$ for 3 weeks in group 2 and for 5 weeks in group 3. Both $10 \% \mathrm{w} / \mathrm{v}$ fipronil-based spot-on solutions, despite different vehicles, were equally able to eradicate tick infestation, to prevent new infestations and were equally well tolerated.

\section{Introduction}

Hard-bodied ticks (family Ixodidae) are temporary parasites that can transmit major systemic diseases as well as being a local cause of inflammation and necrosis (Baxter et al. 2009). The frequency of vector-borne diseases in pets (canine babesiosis, granulocytic anaplasmosis, canine monocytic ehrlichiosis, thrombocytic anaplasmosis) is increasing worldwide (Beugnet and Marie 2009; Fritz 2009). Ixodes ricinus, known as the sheep tick or castor bean tick, is common in Europe (Lindgren et al. 2000; Gray et al. 2009). In dogs, I. ricinus can be a vector of Borrelia burgdorferi (Lyme disease) and Anaplasma phagocytophilum (canine granulocytic anaplasmosis) (Greene 2006). All stages of I. ricinus feed on humans, being able to transmit several diseases to people, including Crimean-Congo hemorrhagic fever and Lyme disease. Therefore, effective tick control in dogs will help preventing canine diseases and may indirectly contribute to prevent human diseases.

First available as a $0.25 \%$ spray, fipronil was then marketed as a spot-on formulation (Postal et al. 1997; Cruthers et al. 2001) and eventually it was combined with $S$-methoprene (Young et al. 2003), also in a spot-on. More recently, pyriprole from the same chemical group had been launched and is available as a spot-on (Schuele et al. 2008). Finally, as fipronil's patent has recently expired, 
new fipronil-based products are now present on the market.

The present study was conducted to evaluate the immediate and sustained efficacy and the tolerance of a new spot-on formulation (Effipro ${ }^{\circledR}$ Spot-on, Virbac S.A.) with the same qualitative and quantitative composition in terms of active ingredient (fipronil) as the original product (Frontline ${ }^{\circledR}$ Top spot, Merial) but with different vehicles. The efficacy was evaluated against experimental infestations of $I$. ricinus in dogs. A positive reference control group included dogs treated with the original product.

\section{Materials and methods}

\section{Animals}

Eighteen (nine female and nine male) Beagle dogs between 11 and 24 months old and weighing from 8.6 to $15.2 \mathrm{~kg}$ were included in the study. Dogs were allocated randomly into three groups of six and each dog was singly housed in a $3 \times 1.4-\mathrm{m}$ pen. Saw dust was used as bedding in each pen. Petroleum jelly barriers were placed around the pen perimeter to prevent ticks from escaping from the pens. Dogs were fed a standard dry commercial dog diet and water was available ad libitum. They were acclimatized for 7 days prior to treatment.

Ticks and experimental infestations

Laboratory-reared adult I. ricinus with a male-female ratio of 40:60 were obtained from Prof. Matuschka, Medizinische Falkultät der Humboldt Universität zu Berlin Abt. Parasitologie, Berlin, Germany. For each experimental infestation, approximately 50 viable unfed ticks $(30 \pm 2$ females and $20 \pm 2$ males) were applied to the dog. For the infestations, dogs were sedated with ketamine $(10 \mathrm{mg} / \mathrm{kg})$ combined with xylazine $(2 \mathrm{mg} / \mathrm{kg})$, both given intramuscularly. Once sufficiently sedated, each dog was infested in its pen; ticks were applied gently to the dorsal or lateral rump area and allowed to crawl into the hair coat. Dogs were infested 7 and 2 days prior to the treatments and 7, 14, 21, 28 and 35 days after the treatments.

\section{Treatments}

The study was of a parallel-arm, randomized block design, single site and blinded controlled. The animals were not treated by an individual involved in the post-treatment assessments and observations. Study groups were coded by colour to blind the staff performing post-treatment assessments and observations. All animals were listed according to their sex and within sex; animals were ranked from the lowest to the highest study day -7 body weight. Where animals with equal study day -7 body weight occurred, they were ranked in order of decreasing animal number. Within each sex, the animals were divided into blocks of three, with one dog in each block assigned to each group using random order numbers derived from Fisher and Yates tables.

The dogs in group 1 were not treated. The dogs in group 2 were treated with a $10 \% \mathrm{w} / \mathrm{v}$ fipronil-based spot-on solution (Frontline ${ }^{\circledR}$ Spot-on Dog, Merial) at a dosage of $0.67 \mathrm{~mL}$ for a dog weighing from 2 to $10 \mathrm{~kg}$ and $1.34 \mathrm{~mL}$ for a dog weighing from 10.1 to $20 \mathrm{~kg}$. The dogs in group 3 were treated with the new fipronil spot-on (Effipro ${ }^{\circledR}$ Spoton, Virbac SA) at an identical dosage $(0.67 \mathrm{~mL}$ for a dog weighing from 2 to $10 \mathrm{~kg}$ and $1.34 \mathrm{~mL}$ for a dog weighing from 10.1 to $20 \mathrm{~kg}$ ). The solution used in group 3 had the same qualitative and quantitative composition in terms of active ingredient (fipronil) as Frontline ${ }^{\circledR}$ Spot-on Dog but some vehicles were different. The treatment took place in the pens in which the dogs were housed. The coat was parted between the shoulder blades until the skin was visible. The tip of the pipette was placed on the skin and gently squeezed at one or two spots to empty its content on the skin. Care was taken when applying the products to reduce the chance of run-off. There was no run-off. Gloves and protective clothing were changed between groups during the study procedures to avoid cross-contamination. The pipette application design (commercially used pipette volume per body weight range and pipette application) was preferred to come closer to the field situation. Application was performed by a well-trained person, ensuring that the pipette was completely emptied.

Concurrent treatments that may have had an antiparasitic activity (e.g. any endo- or ecto-parasiticide) were not allowed.

\section{Clinical examinations and tick counting}

General health observations were carried out on all dogs once daily by a trained technician from day -7 to 37 ; when, in the technician's opinion, the health of a dog was abnormal, a veterinary surgeon was contacted. Dogs were weighed prior to feeding on days $-7,3,11,25$ and 37. Additionally, each animal was submitted to a full clinical examination and skin examination of the administration site prior to the administration of the products and 1, 3, 6, 24 and $48 \mathrm{~h}$ after dosing. Behaviour, salivation, pupil constriction and the presence of nervous signs were assessed and scored from 0 to 3 :

1. Behaviour-0: normal behaviour, 1: dull, 2: head down and not alert, 3: lethargic/moribund

2. Salivation-0: normal salivation, 1: slightly excessive salivation, 2: excessive salivation, 3: profuse salivation

3. Pupil constriction-0: normal, 1: slightly constricted pupil, 2: constricted pupil 
4. Nervous signs - 0: normal behaviour, 1: slight excitability and uncoordinated, 2: very excitable and uncoordinated, 3: lateral recumbency and convulsions.

The skin at the administration site was assessed for cosmetic changes, alopecia, erythema and edema. Any cosmetic changes (e.g. clumping, matting, discoloration) were recorded.

Forty-eight hours after the treatment and 48 hours after each challenge (days-5, 2, 9, 16, 23, 30 and 37), the population of remaining ticks was assessed for each animal. To facilitate counting, the dogs were sedated with the same aforementioned protocol. The dog was then removed from its pen and placed on a table for the evaluation procedure. The hair was pushed manually, using the thumb, fingers or forceps, against its natural lap such that the skin and ticks were exposed. The examiner(s) systematically examined the head, all dorsal and ventral areas and the legs of the dog. On day -5 , the number of live attached ticks were counted and recorded and all ticks were removed. On all other tick counts, the number of live attached and free (on dogs) ticks were quantified and all ticks were removed from the dog.

\section{Statistical analysis}

Original data were entered in an Excel 2000 (Microsoft ${ }^{\circledR}$ Office, Microsoft ${ }^{\circledR}$ Corporation) file and $100 \%$ verification to the original data was performed. The Excel files were imported into Minitab version 14 (Minitab SARL, France), which was the software used for the statistical analysis. For all analyses, the significance threshold was $\alpha=0.05$. The three groups were described and compared before treatment (baseline) on the following criteria: weight, age, pre-study day-5 tick attachment rates and product application rate ( $\mathrm{mg}$ fipronil/kg body weight). Parameters were analysed using a Kruskal-Wallis test or one-way ANOVA (body weight). Arithmetic and geometric means were calculated for each of the three groups at each time point. For groups 2 and 3, efficacy was calculated at each time point using the mean according to the following formula:

$\operatorname{Efficacy}(\%)=100 \times\left(\operatorname{mean}_{\text {control }}-\right.$ mean $\left._{\text {treated }}\right) /$ mean $_{\text {control }}$

An effective dose was expected to provide $>90 \%$ reduction in tick counts compared to control. In addition, tick counts were compared for all groups using KruskalWallis test. When there was a significant difference, the individual groups were compared using Mann-Whitney or Kruskal-Wallis test. If one group contained all zero counts, Kruskal-Wallis test was used instead. In cases where data sets contained a zero, geometric means were calculated by adding 1 to all of the numbers. After the mean was calculated, 1 was subtracted.

\section{Results}

Blinding of the study was not broken. The three groups were homogenous at baseline on the following criteria: age, weight, sex, pre-treatment tick attachment rate and product application rate (mg fipronil/kg body weight) (Table 1). No adverse events were recorded in any of the treatment groups that could be related to the administration of either product.

All animals of group 2 (Frontline ${ }^{\circledR}$ ) and group 3 (Effipro $\left.{ }^{\circledR}\right)$ had clumping of the hairs at the application site at 1,3 and $6 \mathrm{~h}$ post-application. Greasiness of the hair was observed at the application site in all animals of groups 2 and 3,1 and $3 \mathrm{~h}$ after the treatment. Three dogs treated with Frontline ${ }^{\circledR}$ had greasiness of the hair 6 hours post-dosing. Two dogs which had received Effipro ${ }^{\circledR}$ showed hair discoloration and white deposit on the application site $6 \mathrm{~h}$ after application. One dog assigned to the Effipro ${ }^{\circledR}$ group had white deposit on the hair on the application site $24 \mathrm{~h}$ after the application.

The arithmetic mean numbers of ticks that were present in the hair coat of the untreated control dogs and on treated animals $48 \mathrm{~h}$ after each infestation are graphically illustrated in Fig. 1. The efficacy, based on geometric means, of both formulations of fipronil is summarized in Table 2.

The results (Fig. 1; Table 2) show that the experimental infestations with $I$. ricinus were successful, with a mean percentage recovery of I. ricinus on the control dogs $48 \mathrm{~h}$ after each infestation ranging between $37 \%$ (day 23) and $50.7 \%$ (day 9). Efficacy, based on tick reduction, was $>90 \%$ in dogs treated with Frontline ${ }^{\circledR}$ on days 2, 9, 16 and 23 and was $>90 \%$ in dogs treated with Effipro ${ }^{\circledR}$ on all study days, i.e. $2,9,16,23,30$ and 37 .

The differences in tick counts between the two groups treated with a fipronil solution (groups 2 and 3) and the control group (group 1) were significant on all study days $(p<0.01)$. On all study days, tick counts in group 2 (Frontline ${ }^{\circledR}$ ) and group 3 (Effipro ${ }^{\circledR}$ ) were not significantly different.

\section{Discussion}

The recovery rates of at least $37 \%$ at all time points indicate that the strain was sufficiently vigorous and that the dogs used for the trial were adequately susceptible to Ixodes infestation.

Both Effipro ${ }^{\circledR}$ and Frontline ${ }^{\circledR}$ formulations evaluated in this study were effective in treating I. ricinus 
Table 1 Means (standard deviation; $n=6$ ), median, minimal and maximal values of age, weight, pre-treatment tick attachment rate and application rate from dogs treated with one of the two $10 \% \mathrm{w} / \mathrm{v}$ fipronil-based spot-on solutions or left untreated

\begin{tabular}{|c|c|c|c|c|}
\hline Variable & Treatment group & Mean (standard deviation) & Median & Min-max \\
\hline \multirow[t]{3}{*}{ Age (months) } & Negative control & $13.8(2.40)$ & 13.0 & $12.0-18.0$ \\
\hline & Frontline $^{(\mathbb{R}}$ & $15.5(1.76)$ & 15.0 & $14.0-18.0$ \\
\hline & Effipro $^{\circledR}$ & $13.7(5.47)$ & 12.5 & $8.0-24.0$ \\
\hline \multirow[t]{3}{*}{ Weight $(\mathrm{kg})$} & Negative control & $11.8(2.13)$ & 11.2 & $9.5-15.2$ \\
\hline & Frontline $^{\mathbb{R}}$ & $11.5(2.07)$ & 10.6 & $9.6-15.0$ \\
\hline & Effipro $^{\circledR}$ & $11.7(2.35)$ & 11.3 & $8.6-15.0$ \\
\hline \multirow[t]{3}{*}{ Pre-treatment tick attachment rate $(\%)$} & Negative control & $62.2(11.5)$ & 60.0 & $50.0-76.7$ \\
\hline & Frontline $^{(\mathbb{R}}$ & $63.3(14.0)$ & 63.3 & $46.7-83.3$ \\
\hline & Effipro $^{\circledR}$ & $65.0(16.4)$ & 65.0 & $43.3-90.0$ \\
\hline \multirow[t]{2}{*}{ Application rate (fipronil, mg/kg BW) } & Frontline ${ }^{\circledR}$ & $10.6(2.52)$ & 11.1 & $6.7-13.1$ \\
\hline & Effipro $^{\circledR}$ & $10.4(1.97)$ & 10.4 & $7.8-12.9$ \\
\hline
\end{tabular}

infestations with an efficacy of $94 \%$ and $99 \%$, respectively. The two formulations of fipronil prevented new infestations for 5 weeks (Effipro ${ }^{\circledR}$ ) and 3 weeks (Frontline ${ }^{\circledR}$ ), with an efficacy of $>90 \%$ calculated at 48 h postchallenge. The results obtained with the Frontline ${ }^{\circledR}$ formulation were comparable with those obtained in previous studies, where efficacy assessed at $48 \mathrm{~h}$ after a tick challenge was $>90 \%$ for 4 weeks against $I$. ricinus (Pollmeier et al. 2001) and against I. scapularis (Dryden et al. 2006).

The primary concern about tick is their ability to serve as vectors of diseases. B. burgdorferi, the causal agent of Lyme disease, is predominantly transmitted by Ixodes ticks. The maximum transmission of the spirochete occurs between 48 and $72 \mathrm{~h}$ after the nymph attachment (des Vignes et al. 2001; Piesman and Dolan 2002; Greene 2006). Similarly, transmission of the human granulocytic ehrlichiosis agent, A. phagocytophilum (formerly Ehrlichia

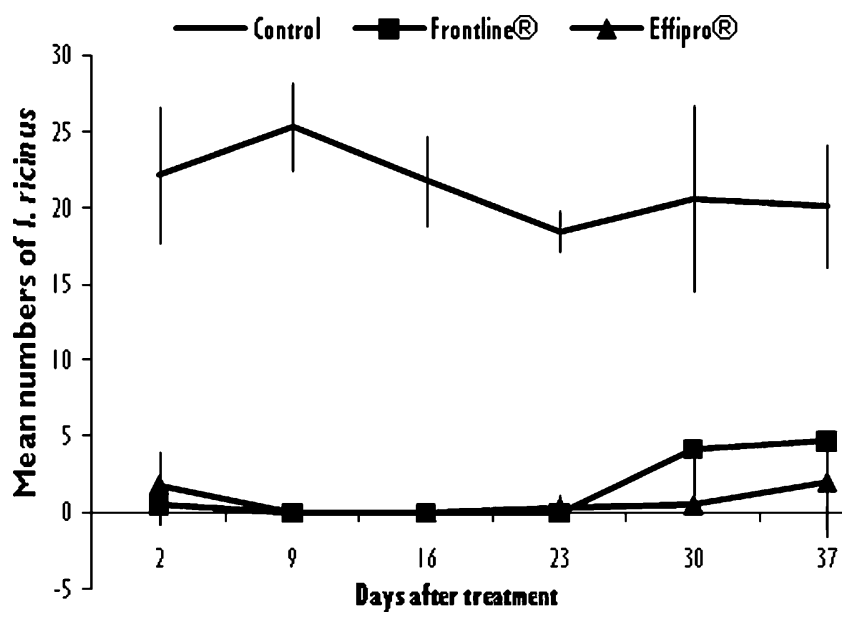

Fig. 1 Arithmetic mean (SD) of I. ricinus counts $48 \mathrm{~h}$ after treatment with two $10 \% \mathrm{w} / \mathrm{v}$ fipronil-based spot-on solutions on day 0 and $48 \mathrm{~h}$ after each re-infestation with 50 young adult ticks phagocytophyla), was estimated to require at least $30 \mathrm{~h}$ in an experimental mice model infested with Ixodes nymphs. Transmission by adults may occur later based on the fact that adult Ixodes complete their blood meal in 7-9 days compared with 3-4 days for nymphs (Katavolos et al. 1998).

Therefore, a killing effect obtained $48 \mathrm{~h}$ after the infestation, such as in the present study, ensures an adequate protection against Lyme disease agents and likely protects against $A$. phagocytophilum.

Both products were well tolerated by all the animals who received the treatments. Only cosmetic changes (greasiness, clumping of the hair coat, white deposit) were seen at the application site of both formulations; they resolved by $24-48 \mathrm{~h}$.

Both Effipro ${ }^{\circledR}$ Spot-on and Frontline ${ }^{\circledR}$ Top spot are $10 \% \mathrm{w} / \mathrm{v}$ fipronil-based spot-on solutions, but some of their vehicles are different. The present study shows that, despite different vehicles, the two formulations provided a similar protection against I. ricinus infestation and that they were equally well tolerated. These results are in agreement with those previously obtained in a study conducted in a similar manner on dogs experimentally infested with fleas. Both $10 \% \mathrm{w} / v$ fipronil-based spot-ons were equally able to eradicate flea infestation, to prevent new infestations and were equally well tolerated. Geo-

Table 2 Mean geometric efficacy (\%) of two 10\% $w / v$ fipronil-based spot-on solutions applied to dogs experimentally infested with I. ricinus, calculated $48 \mathrm{~h}$ after the treatment and $48 \mathrm{~h}$ after each re-infestation

\begin{tabular}{|c|c|c|c|c|c|c|}
\hline & \multicolumn{6}{|c|}{ Days after treatment } \\
\hline & 2 & 9 & 16 & 23 & 30 & 37 \\
\hline Frontline ${ }^{\circledR}$ & 98.8 & 100.0 & 100.0 & 100.0 & 86.3 & 77.2 \\
\hline Effipro $^{\circledR}$ & 93.8 & 100.0 & 100.0 & 98.9 & 97.9 & 94.1 \\
\hline
\end{tabular}


metric mean flea counts obtained were reduced by $99.7 \%$ and $100 \%$ in the groups treated with Effipro ${ }^{\circledR}$ Spot-on and Frontline ${ }^{\circledR}$ Top spot, respectively, on day 2, compared to the negative control group. Dogs were protected from reinfestations with an efficacy of $>95 \%$ for 93 days in the group treated with Effipro ${ }^{\circledR}$ Spot-on and for 79 days in the group treated with Frontline ${ }^{\circledR}$ Top spot (Bonneau et al. 2010).

Open Access This article is distributed under the terms of the Creative Commons Attribution Noncommercial License which permits any noncommercial use, distribution, and reproduction in any medium, provided the original author(s) and source are credited.

\section{References}

Baxter C, Vogelnest L, Doggett S (2009) Dermatoses caused by infestations of immature Ixodes spp. on dogs and cats in Sydney, Australia. Aust Vet J 87:182-187

Beugnet F, Marie JL (2009) Emerging arthropod-borne diseases of companion animals in Europe. Vet Parasitol 163:298-305

Bonneau S, Fourier JJ, Rousseau C, Cadiergues MC (2010) Comparative efficacy of two fipronil spot-on formulations against experimental flea infestations (Ctenocephalides felis) in dogs. Intern J Appl Res Vet Med 8:16-20

Cruthers L, Slone RL, Guerrero AJ, Robertson-Plouch C (2001) Evaluation of the speed of kill of fleas and ticks with frontline top spot in dogs. Vet Ther 2:170-174

des Vignes F, Piesman J, Heffernan R, Schulze T, Stafford K III, Fish D (2001) Effect of tick removal on transmission of Borrelia burgdorferi and Ehrlichia phagocytophila by Ixodes scapularis nymps. J Infect Dis 183:773-778

Dryden MW, Payne PA, Smith V, Hosteder J (2006) Evaluation of an imidacloprid $(8.8 \% \mathrm{w} / \mathrm{w})$-permethrin $(44.0 \% \mathrm{w} / \mathrm{w})$ topical spot-on and a fipronil $(9.8 \% \mathrm{w} / \mathrm{w})-(\mathrm{S})$-methoprene $(8.8 \% \mathrm{w} / \mathrm{w})$ topical spot- on to repel, prevent attachment, and kill adult Ixodes scapularis and Amblyomma americanum ticks on dogs. Vet Ther 7:173-186

Fritz CL (2009) Emerging tick-borne diseases. Vet Clin N Am Small Anim Pract 39:265-278

Gray JS, Dautel H, Estrada-Pena A, Kahl O, Lindgren E (2009) Effects of climate change on ticks and tick-borne diseases in Europe. Interdiscip Perspect Infect Dis 2009:593232

Greene C (2006) Environmental factors in infectious disease. In: Greene C (ed) Infectious diseases of the dog and cat. Saunders Elsevier, St Louis, MI, pp 991-1012

Katavolos P, Armstrong P, Dawson J, Telford S III (1998) Duration of tick attachment required for transmission of granulocytic ehrlichiosis. J Infect Dis 177:1422-1425

Lindgren E, Talleklint L, Polfeldt T (2000) Impact of climatic change on the northern latitude limit and population density of the disease-transmitting European tick Ixodes ricinus. Environ Health Perspect 108:119-123

Piesman J, Dolan M (2002) Protection against Lyme disease spirochete transmission provided by prompt removal of nymphal Ixodes scapularis (Acari: Ixodidae). J Med Entomol 39:509-512

Pollmeier M, Pengo G, Jeannin P, Soll MD (2001) Evaluation of the efficacy of FRONTLINE (R) (active ingredient: Fipronil) in the treatment and control of European castor bean tick (Ixodes ricinus) infestations in the dog and cat. Kleintierpraxis 46:99-104

Postal JM, Longo F, Jeannin PC, Hunter JS, Tanner P, Keister DM, Romano M (1997) Efficacy against ticks (Rhipicephalus sanguineus) in dogs of two topical formulations of fipronil (Frontline spray and Frontline Top-Spot) after experimental infestation: an overall analysis of various studies. Fourth International Symposium on Ectoparasites of Pets. N. C. Hinkle. University of California, Riverside, CA, USA, pp 105-106

Schuele G, Barnett S, Bapst B, Cavaliero T, Luempert L, Strehlau G, Young DR, Moran C, Junquera P (2008) Efficacy of a single application of a $12.5 \%$ pyriprole topical solution against laboratory infestations with ticks (Ixodes ricinus, Dermacentor reticulatus, and Rhipicephalus sanguineus) on dogs. Vet Parasitol 154:311-317

Young DR, Arther RG, Davis WL (2003) Evaluation of K9 advantix (TM) vs. frontline plus (R) topical treatments to repel brown dog ticks (Rhipicephalus sanguineus) on dogs. Parasitol Res 90: S116-S118 